\title{
Development of a Computer Program for Volume Estimation of Arteriovenous Malformations from Biplane DSA Images
}

\author{
Roger Lundqvist ${ }^{1}$, Michael Söderman ${ }^{2}$, Kaj Ericson ${ }^{2}$, Ewert Bengtsson ${ }^{1}$, and \\ Lennart Thurfjell ${ }^{1}$ \\ 1 Centre for Image Analysis, Uppsala University, \\ Uppsala, Sweden \\ 2 Dept. of Neuroradiology, Karolinska Hospital \\ Stockholm, Sweden
}

\begin{abstract}
The goal of this work was to develop a computer program for accurate estimation of size and location of intracranial arteriovenous malformations (AVM) from biplane digital subtraction angiography (DSA) images. The program will be used for diagnostic purposes to predict the outcome of Gamma knife radiosurgery and thus give basic data for the optimization of the management and comparison of different treatment modalities. Our solution is based on the so called intersecting cone model (ICM). Volume measurements with this model have been shown to correlate well to volume data from the dose-planning equipment (Leksell GammaPlan $\AA$, Elekta, Sweden). The method described in this paper produces a fast and accurate implementation of the ICM suitable for clinical practice. The implementation has been validated from phantom images and simulated images and the errors have been shown to be small compared to the approximations made by the ICM.
\end{abstract}

\section{Introduction}

Accurate volume estimation of intracranial arteriovenous malformations (AVMs) is important in radiosurgical treatment. The risk for complications is directly related to the irradiated volume and the radiation dose given, as are the chances of cure [1, 2], 3. In Gamma Knife radiosurgery (GKRS) of AVM, the dose planning (Leksell GammaPlan $\AA$, Elekta, Sweden) is based on biplane digital subtraction angiography (DSA) images, preferably integrated with magnetic resonance imaging (MRI) 4] and 5]. Such dose planning is usually only available in institutions where GKRS is performed. In the clinical situation knowledge about the predicted outcome of GKRS is valuable in patient management, even if the facilities for GKRS are lacking [6]. For example centres performing AVM embolisation may find it valuable to accurately estimate AVM volume from diagnostic angiography before and after procedures, for an objective evaluation of the results. They may also compare their results with the radiosurgical outcome model and optimize the combined treatment [7]. A potentially hazardous continued endovascular treatment may be halted when the volume reduction has 
reached a level where radiosurgery can safely be applied. This assessment may be done by placing a stereotactic box over the head of the patient at the end of the diagnostic angiography and perform volume measurement, [6] and [7].

Some years ago a computer program for measurement of AVM volumes was developed at the Karolinska Hospital, in collaboration with General Electric Medical Systems. It was based on two or more most often perpendicular DSA images, with the AVM contour delineated in each of them. The volume was approximated with the interior of the intersection of the two X-ray cones intersecting the AVM contours in stereotactic space. The approach was named the intersecting cone model (ICM). The values obtained with ICM is an overestimation of the AVM volume, but it has been shown that the difference is small when compared to data from the dose planning [7. The program developed at that stage was, however, closely connected to an old version of the GE Advantage Windows system and not easily applicable for further improvements. The measurement algorithms used internal functions in the GE Advantage Windows system, which made them hard to port to a new program. Finally, the algorithm for the volume measurements was very slow when the sizes of the AVM volumes were large.

These limitations of the old program gave rise to the decision to develop a new program, independent of the old one and with new algorithms to solve the volume measurement problem, but still based on the ICM. The goal was to create a fast and flexible system suitable for clinical practice and further development. The result of this work, including validation and description of the methods used, is presented in this paper.

\section{Methods}

All DSA images used in this work were acquired on a General Electric Medical Systems Advantx $(\mathbb{B}$, angiography equipment. The image file format for all images followed the DICOM 3.0 standard. The DSA images were corrected for geometric distortion by an algorithm developed in collaboration with General Electric Medical Systems [8], before the volume measurement process was started.

\subsection{Stereotactic Box Calculations}

The first step is to register the different DSA projections into the same coordinate system. This is made from a stereotactic plexiglass box (Elekta, Sweden) with engraved fiducials, which is used during the angiography. It is essential that the box is retained in the same position relative to the head during acquisition of the lateral and PA projections, but this is easily achieved with biplane angiography without the need to fix the box to the head of the patient. The detection of the stereotactic box in the images is performed by manual marking of the fiducials by the mouse, see Fig. I

We consider the stereotactic box coordinate system as fixed and the positions of the X-ray origin $X_{0}$ and projection image plane $P_{p}$ as moved relative to 

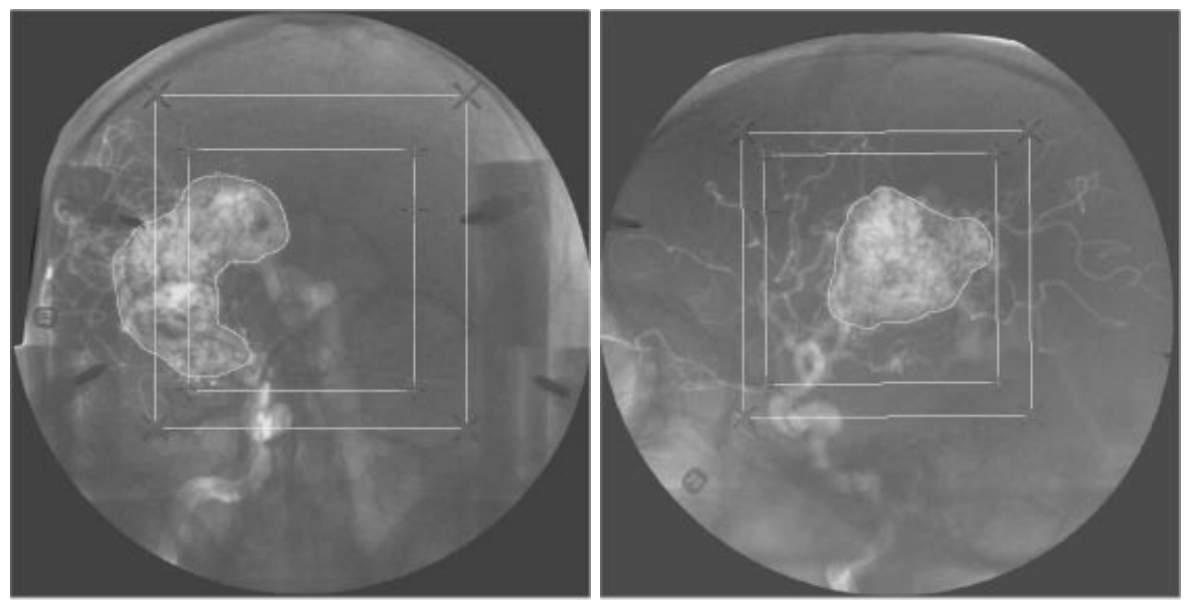

Fig. 1. In the images two different DSA projections with a large visible AVM are shown. The stereotactic box has been defined and the AVM has been delineated by the user

this coordinate system, see Fig. 2. The projection image plane can be located anywhere along the X-ray direction. In our solution, however, we regard it as located at the specific position where the pixel size in the projected image corresponds to some specific predefined value. This allows us to couple distances and positions in the projected 2D-image to positions in stereotactic 3D-space.

We need to know a number of variables to be able to do the registration of the images into stereotactic space. For each projection, the distance $d_{1}$, from the box centre to $X_{0}$, and the distance $d_{2}$, from box centre to $P_{p}$, have to be found. Also the relative rotation around the three box axes of the vector $\overline{v_{3}}$, pointing in the X-ray direction, has to be found. These rotations we denote as $r_{x}, r_{y}$ and $r_{z}$. Finally, we also have to find the translation of the point in the middle of the $P_{p}$ image matrix, which we denote as $p_{0}$, relative to the box centre. This is expressed as a two component translation, $d_{3}$ and $d_{4}$, along the two base-vectors $\overline{v_{1}}$ and $\overline{v_{2}}$ spanning the projection image plane $P_{p}$.

From the three rotations we can build a rotation matrix $\mathbf{R}$. If we denote the base vectors $\overline{b_{1}}, \overline{b_{2}}$ and $\overline{b_{3}}$ in the stereotactic space, which are the unit vectors in respective direction, we can express the base-vectors in the $X_{0}-P_{p}$ coordinate system according to:

$$
\overline{v_{1}}=R \overline{b_{1}} \quad \overline{v_{2}}=R \overline{b_{2}} \quad \overline{v_{3}}=R \overline{b_{3}}
$$

This leads to the following equations for the points $X_{0}$ and $P_{p}$ :

$$
\overline{X_{0}}=-d_{1} \overline{v_{3}}+d_{4} \overline{v_{1}}+d_{3} \overline{v_{2}} \quad \overline{p_{0}}=d_{2} \overline{v_{3}}+d_{4} \overline{v_{1}}+d_{3} \overline{v_{2}}
$$



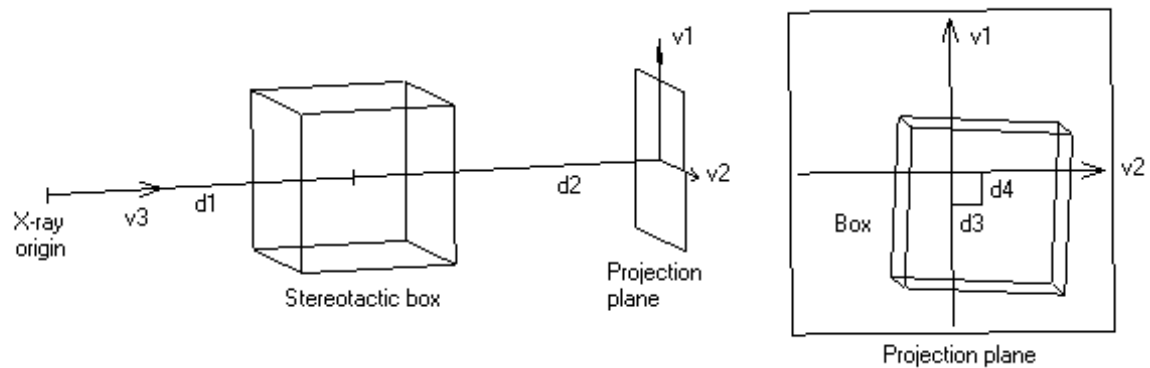

Fig. 2. The left part of the figure shows a sideview of the angiography examination. To the right we see the same scene viewed in the direction from the X-ray source in direction of the point in the middle of the projection image matrix

If we use the cosinus-theorem it is possible to calculate a projected point $p^{\prime}$ on $P_{p}$ for every 3D-point $p$ in the stereotactic space, since we know the basevectors and origins for the two coordinate systems. In the same way it is possible to express the equation for an arbitrary ray through $3 \mathrm{D}$-space from point $X_{0}$ to a point $p^{\prime}$ on $P_{p}$.

There are a number of different approaches to find the 7 parameters $\left(r_{x}, r_{y}\right.$, $\left.r_{z}, d_{1}, d_{2}, d_{3}, d_{4}\right)$ needed to perform these calculations. We have chosen to solve this problem by using a numerical method, since an analytical approach would lead to an overdetermined equation system and still result in an approximation of the real solution. We use an iterative optimization method based on Powell's algorithm implemented as it is described in [9], where a cost function expressing how well a specific parameter set solves the projection problem is minimized. In our solution this cost function calculates the sum of the distances between the box fiducials projected down on $P_{p}$ and the corresponding points marked by the user directly in the image. For each projection there are totally eight fiducials used. The optimization process results in one optimal parameter configuration for each projection image which defines the images relation to the stereotactic space.

\subsection{Volume Measurement Calculations}

The next step is to draw the AVM contours in at least two DSA projections by the mouse, see Fig. 1. After that a result volume is created in the computer memory to be used when calculating the AVM volume. The center of it corresponds to the middle of the stereotactic coordinate system. In the next step, rays are cast through this volume from $X_{0}$ to all points lying on the drawn AVM contour on $P_{p}$. All voxels passed by the rays are set to a non-zero value in the result volume. This results in a hollow cone for each projection, which is filled through a floodfill algorithm. A different bit is set for each projection, which makes it possible to find the voxels that are set in both projections. In the next step the number of 
voxels set in both projections are counted and multiplied by the volume of an individual voxel to find an estimation of the total AVM volume.

There is a problem if we want to use the full image resolution in this process, since typically DSA images have a matrix of $1024 x 1024$, leading to a result volume of a size in the order of $1024 \times 1024 \times 1024$ voxels. Even if only 2 data bits per voxel is enough to handle the storage of the resulting volume, it would still be a too large volume for most computers. The solution to this is to perform the volume measurement in a two step multi-resolution process. First we use a low resolution result volume, $128 \times 128 \times 128$ voxels, and perform the whole process. The boundaries of the AVM volume are found and a new result volume, containing only the part with the AVM, with 8 times higher resolution is created. This results in a volume with manageable size, which is used in the final step where the whole process of casting rays through the AVM contours is repeated once again. It can also be noticed that the edge voxels of the cones are not completely belonging to the AVM. Since the number of such voxels becomes large at the finest resolution, we make the assumption that they contribute with approximately half their size to the measured volume. For this reason the edge voxels are counted separately and only half of their voxel volume are added to the final result.

\section{Experiments}

\subsection{Phantom Data}

Some geometrical object phantoms were constructed and mounted inside the stereotactic box. These were two cylinders of different sizes and one rectangular box shaped object. The objects were measured manually and their "true" volumes were calculated based on their geometrical forms. Two different image series were acquired to test for different positions of the objects. In both series the objects were positioned with their sides in approximately perpendicular angles towards the DSA projections to test the ability of the program to make an accurate volume estimation, Fig. 3. The only difference between the series was a translation of the objects relative to the X-ray sources. The objects were redrawn and processed three times for each series and the results were compared to the true volumes.

\subsection{Simulated Data}

A validation of the combined errors from the manual marking of the stereotactic box together with the numerical solution of the box equation system was also made. A test program was written in $\mathrm{C}++$ using OpenGL to create simulated images of the box fiducials, with known rotation angles, translations and distances relative to the imaging equipment. First the known positions of the fiducials were defined in a 3D coordinate system and then a random configuration for the parameter transformations were chosen within reasonable limits. These 

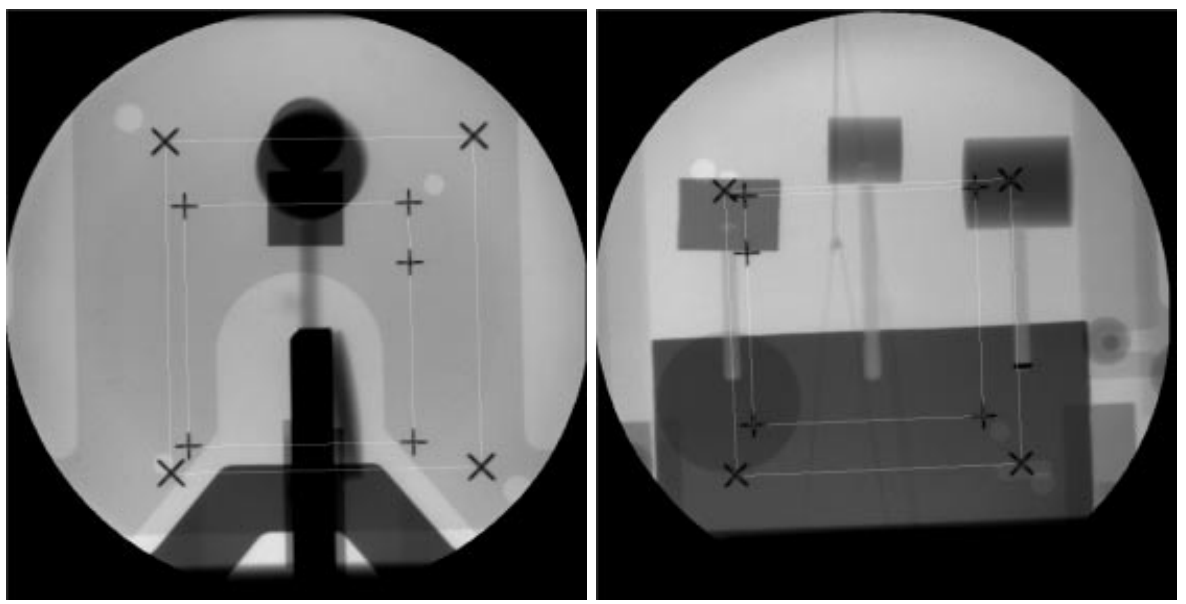

Fig. 3. In this figure two approximately perpendicular projections of the phantom images used in the experiments are shown

transformations were applied to the 3D coordinate system through OpenGL:s internal functions for rotations, translations and perspective projection and the fiducials were drawn in 3D space and projected onto the image plane, Fig. 4.

After this procedure the image was saved and loaded into the volume measurement program and processed in the same way as the real DSA images, hence resulting in an estimated solution of the true parameter configuration used when creating the simulated image. A total of 50 simulated images were constructed in this way and processed by the program and maximal, absolute mean and standard deviation for the errors in the transformation parameters were calculated.

Finally, the contribution of these errors on the outcome of the actual volume measurement were studied. This was accomplished by randomly adding errors to the parameters, based on the knowledge of the error size, and compare the volume measurements with parameter errors included to those without. The parameter errors, however, were not independent of each other, since different transformations compensate for errors made by others. For this reason we made an inspection of the distributions of the errors and found that an assumption of a multivariate normal distribution seemed justifiable. According to that assumption the random errors were produced by generating multivariate random numbers based on the covariance matrix of the errors. This was repeated for some AVM volumes of different sizes and for each volume a total of 100 volume measurements with included errors were made. The distribution of these measurements compared to the values without added errors was then examined. 


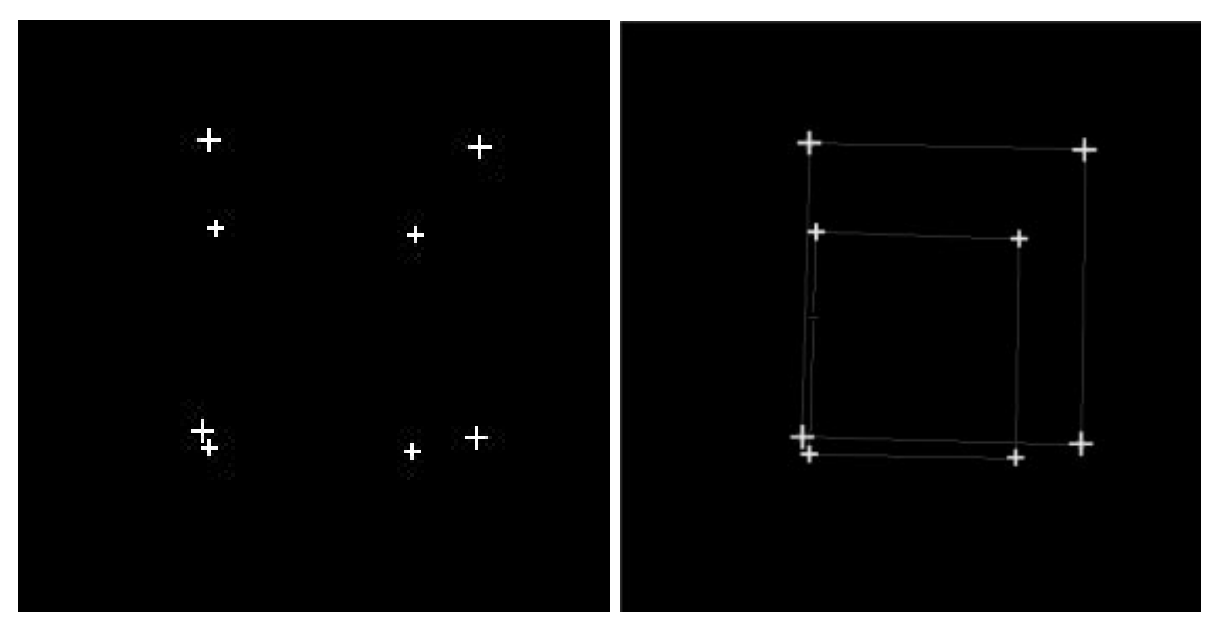

Fig. 4. In this figure a simulated image of the stereotactic fiducials before and after definition of the stereotactic box is shown

\subsection{Comparisons to Old Program}

The old program has been validated earlier against the radiosurgical dose planning system (LGP) and showed good correlation with that 7]. For that reason the volume estimates of the new program also has been compared with results from the old program. A perfect comparison is impossible to accomplish since the file formats for saving the manual marks of the fiducials and drawn AVM contours are not compatible between the systems. This means that the manual interaction has to be performed twice, independently of each other. Nevertheless, a number of measured volumes from different examinations have been compared to verify that the two programs produce similar results.

\section{Results}

\subsection{Phantom Data}

Due to the specific geometrical forms of the phantoms, the measured volumes with the ICM should be very close to the true volumes, provided that they are positioned with their sides at right angles to the two DSA projections. In Table 1 the results from the estimates of the phantom volumes based on the ICM compared to the volumes calculated from the geometrical forms of the objects are presented. In the comparisons we can see that the spread for the same objects within series is small compared to the between series measurements, although the angles of the objects were very similar. Wee can also study the accuracy of the method. For all objects the series which produces the best result gave a very good approximation of the volume. However, we should also notice that the 
measurements are actually smaller than the reference volume for some series. The best explanation to this is that the reference value also is an estimation of the volume since it is based on manual measurements. The edges of the objects were also not perfectly sharp in the images which could lead to some errors when drawing the contours.

Table 1. Volume measurements from phantom images. In each series three measurements were made based on differently drawn AVM contours

\begin{tabular}{lrrrrr}
\hline Object & Ref. value & $\begin{array}{r}\text { Series 1 } \\
\text { Mean }\end{array}$ & $\begin{array}{r}\text { Series 1 } \\
\text { Range }\end{array}$ & $\begin{array}{r}\text { Series 2 } \\
\text { Mean }\end{array}$ & $\begin{array}{r}\text { Series 2 } \\
\text { Range }\end{array}$ \\
\hline Small cylinder & 5.840 & 5.745 & 0.114 & 5.565 & 0.022 \\
Large cylinder & 15.303 & 16.037 & 0.030 & 15.287 & 0.086 \\
Rectangular box & 11.088 & 10.540 & 0.059 & 11.076 & 0.120 \\
\hline
\end{tabular}

\subsection{Simulated Data}

In Table 2 the results from the error estimates of the transformation parameters based on the simulated image experiments are presented. It is clear that the errors are not particularly large except for parameters $d_{1}$ and $d_{2}$. These errors, however, compensate for each other to a large extent and the correlation factor was 0.93 . For this reason the overall error caused by these parameters are small compared to other sources.

Table 2. Distribution of volume measurements when random errors are added. A total of 100 measurements for each volume were made

\begin{tabular}{lrrrrrr}
\hline Measures & No error & Mean & Min & Max & Stdev & Range \\
\hline Volume 1 & 1.230 & 1.228 & 1.183 & 1.257 & 0.015 & 0.074 \\
Volume 2 & 2.190 & 2.205 & 2.153 & 2.303 & 0.031 & 0.150 \\
Volume 3 & 3.162 & 3.154 & 3.129 & 3.187 & 0.011 & 0.058 \\
Volume 4 & 4.564 & 4.552 & 4.421 & 4.616 & 0.029 & 0.196 \\
Volume 5 & 6.238 & 6.232 & 6.135 & 6.328 & 0.037 & 0.193 \\
Volume 6 & 8.383 & 8.385 & 8.265 & 8.471 & 0.034 & 0.206 \\
Volume 7 & 11.815 & 11.835 & 11.707 & 12.062 & 0.077 & 0.355 \\
\hline
\end{tabular}

The results from the study of how the parameter errors effect the volume measurements are presented in Table 3. We can see from this table that the spread of the measurements for the same volume gets larger with larger AVM volume size. However, these deviations should be compared to the size of the 
overestimation error with the ICM. For instance if the AVM has an ellipsoid shape the overestimation is about $20 \%$. This can be compared with the maximal deviation from the mean value in these measurements, which never exceeds $5 \%$. For this reason a conclusion is that the errors made by the program, caused by approximations in the calculations and manual interaction, are not decisive when studying the accuracy of the method.

Table 3. Parameter errors from simulated image experiments. A total of 50 images were used

\begin{tabular}{lrrrrrrr}
\hline Errors & $\mathrm{r}_{x}$ & $\mathrm{r}_{y}$ & $\mathrm{r}_{z}$ & $\mathrm{~d}_{1}$ & $\mathrm{~d}_{2}$ & $\mathrm{~d}_{3}$ & $\mathrm{~d}_{4}$ \\
\hline Abs. mean & 0.108 & 0.096 & 0.130 & 4.837 & 3.983 & 0.124 & 0.115 \\
Stdev & 0.153 & 0.122 & 0.158 & 5.868 & 4.879 & 0.114 & 0.092 \\
Max & 0.381 & 0.308 & 0.371 & 13.873 & 11.606 & 0.366 & 0.299 \\
Range & 0.750 & 0.568 & 0.674 & 26.271 & 19.940 & 0.459 & 0.340 \\
\hline
\end{tabular}

\subsection{Comparisons to Old Program}

The volume estimates calculated with the new program are in agreement with the old program. Some minor differences can be noticed, which can be explained by the fact that the manual interaction is performed independently two times. The differences in results are within the error range found from the simulated image experiments and they are also very small compared to the assumptions made by the ICM.

The processing time with the new program is much shorter than the old program. For example a calculation of a large AVM takes less than 5 seconds with the new program using a PC with an Intel Pentium III 500MHz CPU. A similar volume could take several minutes to calculate with the old program. Even though the computers are not directly comparable a significant improvement in performance can be noticed with the new algorithms, which makes the program much more suitable for clinical use.

\section{Discussion and Conclusions}

We have developed a computer program for estimation of AVM volumes based on biplane DSA images. The program has been thoroughly validated against phantom data and simulated images to estimate the errors in the volume measurement. It has been clearly shown that the errors made by using the program are not large compared to the assumptions of the ICM. The implementation is also fast which makes it very useful for clinical practice. The system will be extended with improved functionality for image database support and there are 
several clinical applications for it. One is the possibility to calculate already at the diagnostic angiography and decide if a newly detected AVM is suitable for radiosurgical treatment. An early decision may then be taken to treat the patient with endovascular means or open surgery in the first place. A repeated volume measurement after this treatment will show if the AVM now is treatable with radiosurgery. An obvious application is, of course, as quality control of endovascular procedures. The results of embolisations are usually estimated in very vague terms. A simple and relatively accurate volume measurement will objectively show how much that has been accomplished with embolisation.

\section{Acknowledgments}

This project was funded by the Swedish Foundation for Strategic Research through the VISIT-program.

\section{References}

1. Karlsson B., Lax I., Söderman M.: "Factors influencing the risk for complications following Gamma Knife radiosurgery of cerebral arteriovenous malformations", Radiotherapy and Oncology 1997;43(3):275-80

2. Karlsson B., Lax I., Söderman M.: "Can the probability for obliteration of arteriovenous malformations following radiosurgery be accurately predicted?", Int Journ Rad Onc Biol Phys 1999;43(2):313-319

3. Karlsson B., Lax I., Söderman M.: "Risk for haemorrage in the latency period following Gamma Knife radiosurgery for cerebral arteriovenous malformations", Int. J. Rad. Onc. Biol. Phys. 2000;Submitted

4. Guo W., Lindqvist M., Lindqvist C., et al.: "Stereotaxic Angiography in Gamma Knife Radiosurgery of Intracranial Arteriovenous Malformations", AJNR 1992;13:1107-1114

5. Guo W-Y., Nordell B., Karlsson B., et al.: "Target delineation in radiosurgery for cerebral arteriovenous malformations. Assessment of the value of stereotaxic MR imaging and MR angiography", Acta Radiol 1993;34(5):457-63

6. Ericson K., Söderman M., Karlsson B. and Lindquist C.: "Volume determination of intracranial arteriovenous malformations prior to stereotactic radiosurgical treatment", Interventional Neuroradiology 2 1996;271-275

7. Söderman M., Karlsson B., Launnay L., Thuresson B. and Ericson K.: "Volume measurement of cerebral arteriovenous malformations from angiography", Neuroradiology 2000, In press.

8. Söderman M., Picard C. and Ericson K.: "An algorithm for correction of distortion in stereotactic digital subtraction angiography", Neuroradiology 40: 1998;277-282

9. Press W.H., Flannery B.P., Teukolsky S.A., Vetterling W.T.: "Numerical recipes in C", Cambridge: Cambridge University Press, 1988. 